\begin{tabular}{ccc}
\hline & \\
& International Journal of Case Reports \\
(ISSN:2572-8776)
\end{tabular}

\title{
Recognizing Traditional Chinese Medicine Customs during the COVID-19 Pandemic: A Case Report
}

\begin{abstract}
Justin Chin ${ }^{1,2}$, Christine Lomiguen ${ }^{2,3}$, Kevin Hon ${ }^{4}$, George Nguyen ${ }^{5}$, Cynthia Carmichael ${ }^{1}$
${ }^{1}$ Department of Family Medicine, Lifelong Medical Care, CA USA; ${ }^{2}$ Department of Medical Education, Lake Erie College of Osteopathic Medicine, PA USA; ${ }^{3}$ Department of Family Medicine,

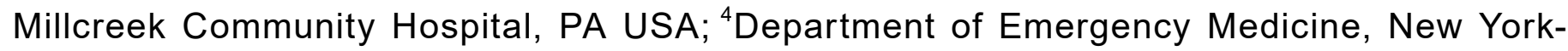
Presbyterian Queens Hospital, NY USA; ${ }^{5}$ Department of Internal Medicine, Banner University Medical Center - Phoenix, AZ USA
\end{abstract}

\section{ABSTRACT}

Traditional Chinese Medicine (TCM) is based on the belief of vital energy (qi) within the body and attributes disease with imbalance in energies (yin and yang). The COVID-19 pandemic has necessitated unique physical barriers, including increased physical distancing, use of personal protective equipment (PPE), and telehealth services, which complicate existing efforts to understand alternative health practices. This case report highlights the importance of cultural competency training for healthcare providers, as evident in improved patient care when recognizing and acknowledging TCM customs in association with the COVID-19 pandemic.

Keywords: Traditional Chinese Medicine Customs; COVID-19 Pandemic; Case Report
*Correspondence to Author:

Justin Chin ${ }^{1,2}$

${ }^{1}$ Department of Family Medicine, Lifelong Medical Care, CA USA; ${ }^{2}$ Department of Medical Education, Lake Erie College of Osteopathic Medicine, PA USA

How to cite this article:

Justin Chin, Christine Lomiguen, Kevin Hon, George Nguyen, Cynthia Carmichael. Recognizing Traditional Chinese Medicine Customs during the COVID-19 Pandemic: A Case Report . International Journal of Case Reports, 2021,5:239.

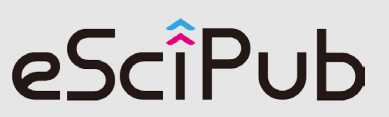

eSciPub LLC, Houston, TX USA. Website: http://escipub.com/ 


\section{Introduction:}

Traditional Chinese Medicine (TCM) traces its roots to China in the $14^{\text {th }}-11^{\text {th }}$ century, before the Common Era (BCE), during the Shang dynasty and has since propagated across the East Asian cultural sphere due to immigration and establishment of diasporas [1]. Based on the belief of vital energy (qi) within the body, TCM attributes disease processes with imbalance in energies (yin and yang) ${ }^{[2]}$. Treatment in TCM requires visual recognition of disease patterns and subsequent alteration of food and lifestyle to restore bodily balance. In comparison, Western medicine focuses more on diagnosis through empirical measures such as imaging and blood work, with disease management accomplished through single-molecule pharmaceuticals. These diagnostic and treatment approach variations present unique challenges for patients and healthcare providers alike when interacting for the first time, often resulting in miscommunication, misdiagnosis, and mistreatment ${ }^{[3,4]}$.

The COVID-19 pandemic has further complicated efforts to understand alternative health practices as cultural or colloquial descriptions of symptoms can be misconstrued as some of the non-specific general symptoms of the disease ${ }^{[5]}$. Due to the highly contagious and relatively asymptomatic spread of the severe acute respiratory syndrome coronavirus 2 (SARS-CoV-2), containment of COVID-19 community spread has relied on social distancing, personal protective equipment, and contact tracing with positive testing ${ }^{[5,6]}$. While several vaccines have since been approved for emergency use in early 2021, it is likely that COVID-19 will have a substantial impact on healthcare for many years to come.

Here we present a case of gastroesophageal reflux that was initially diagnosed as COVID-19 rule out in a Mandarin-speaking patient. In particular, cultural competency and TCM practices will be discussed in relation to Western medicine during the COVID-19 pandemic.

\section{Case Presentation}

Ms. $\mathrm{K}$ is a 56 -year-old Mandarin speaking only female who initially presented to the family medicine clinic to establish care. She had previously seen four other providers and wished to consolidate care under one physician. Her past medical history was significant for hypertension, hyperlipidemia, prediabetes, gastroesophageal reflux, hypothyroidism, was status post total hysterectomy and oophorectomy for uterine cancer over 20 years ago. Medications included hydrochlorothiazide, atorvastatin, levothyroxine, and omeprazole. She was up to date in her health maintenance (mammogram, colonoscopy, and immunizations) and was otherwise healthy.

In her prior primary care visits, she complained of episodes of vague, intermittent chest discomfort that would last several days in duration then self-resolve. Each episode did not have a specific trigger and were accompanied by non-specific symptoms, including general malaise, muscle aches, and fatigue. She denied fever, frank chest pain, shortness of breath, or dyspnea on exertion. During her initial visit to establish care, she noted that her last episode was several weeks prior and that past cardiac workup was negative, however she could not recall the exact diagnosis or where her records were located. Vitals were within normal limits, laboratory orders was unremarkable, and pointof-care electrocardiogram was essentially unchanged from prior workup two years ago. The patient was reassured that her discomfort was likely multifactorial with gastroesophageal reflux and musculoskeletal etiology, with no signs of cardiac pathology.

In the ensuing months, she would call to complain every 1-2 months about return of the chest discomfort with accompanying fatigue and malaise. She noted that she was a food service worker and that she believed that she had an imbalance in her energies, for which she described as "hou qi da or 火氣大”. She noted that she wanted to eat more "cooling foods" to assist in maintaining balance as she was 
recently eating more fried foods. During a triage visit, the aforementioned complaints of fatigue and malaise were construed as symptoms of COVID-19, resulting in the patient obtaining COVID-19 testing, which was ultimately negative. She later returned to the family medicine clinic and asked if her having "huo qi da" would increase the risk of COVID-19 or if it was more related to her gastroesophageal reflux.

In a follow-up telemedicine visit, time was spent understanding the patient's concerns and health beliefs regarding her symptoms, especially as they intersected between TCM and Western medicine. The patient was reassured that her energy imbalances did not place her at further risk for COVID-19 infection and that her plan to change her diet would not only improve her energy balance, but also benefit her hyperlipidemia and reflux symptoms. At her latest visit, she was doing well with no additional complaints.

\section{Discussion}

Chinese food therapy is a series of beliefs and practices centered around the effect of food on the body. Rooted in TCM and Taoist religious theories, numerous iterations exist in Chinese and Chinese diaspora communities, each with their own unique understanding and interpretation [7]. The central tenet of Chinese food therapy is that food and medicine share a common origin, to which food can be used to prevent or treat illness [8]. Taking cues from TCM, food can be classified as "hot/yang", or "cool/yin" and disease states can be triggered when there is imbalance in the body or cured when equilibrium is restored ${ }^{[1,2]}$. While families can have their own variations, traditionally hot foods are those that are cooked at high temperatures or raise body temperature such as ginger, pepper, and garlic [9]. Cold foods are green leafy vegetables, watermelon, and are typically lower calorie items. Over- or underconsumption of foods in either category can lead the body to become weak or fatigued. Literature or empirical research on this topic in
English are limited and difficult to interpret through the lens of randomized controlled trials and objective data ${ }^{[10,11]}$.

Hou qi da (火氣大 in Mandarin Chinese) or yeet hay (熱氣 in Cantonese Chinese) describes a bodily state when there is an abundance of hot/yang energy ${ }^{[12]}$. Patients can report feeling overheated, overstimulated, or tired, which can manifest as skin lesions, heartburn, sore throat, halitosis, and constipation. In these cases, patients will often eat cool/yin foods that will restore balance and bring them out of a hot energy state ${ }^{[13]}$. A common herbal remedy is " 24 flavor tea," a drink that contains ingredients such as chrysanthemum, dried monkfruit, peppermint, and bamboo leaves ${ }^{[14]}$. Resolution of symptoms often occurs in five to seven days after aforementioned dietary and herbal interventions. Translating this to Western medicine, these symptoms can coincide with gastroesophageal or laryngoesophageal reflux, which is similarly treated by diet modifications, but also with medications such as proton pump inhibitors or histamine blockers [15, 16]. A symptom comparison can be found in Table 1. For many Chinese diasporic communities, there is often a blending of Western medicine and TCM as a result of assimilation and enculturation, which can be challenging to inexperienced healthcare providers ${ }^{[17]}$.

Cultural practices in medicine and health can complicate patient care for providers that are unaccustomed to working or understanding these populations ${ }^{[18-20]}$. For example, patients may not report use of herbal remedies or vitamins as they may not consider them pertinent or as medications from a Western medicine point of view. Similarly, healthcare providers may misinterpret or fail to recognize the effects of TCM in relation to Western medicine. It is well-documented that alternative health beliefs and customs can result in adverse or delayed medical care through miscommunication or misinterpretation. With the COVID-19 pandemic, it has become more challenging to engage patients due to infection 
control measures which have limited in-person visits and necessitated telemedicine ${ }^{[5,6]}$. It is unclear whether the aforementioned case would have needed a COVID-19 test if the patient had talked with a physician who understood TCM and correlated that to her COVID-19 risk factors. Greater efforts are needed in medical education to better train physicians to recognize alternative health practices, particularly in the setting of a global pandemic.

\begin{tabular}{l|l} 
Huo Qi Da Symptoms & Gastroesophageal Reflux Symptoms \\
\hline Heartburn/Indigestion & Heartburn/Indigestion \\
\hline Rash & Chest Pain \\
\hline Sore Throat & Sore Throat/Difficulty Swallowing \\
\hline Dry Skin & Globus Sensation in Throat \\
\hline Halitosis & Chronic Cough \\
\hline Constipation & Regurgitation of Food/Acid
\end{tabular}

Table 1: Comparison of TCM “Huo Qi Da” versus Gastroesophageal Reflux Symptoms

\section{Conclusion}

Treatment of disease processes through TCM often involves the use of "cool" and "hot" foods to restore vital energy balance "yin" and "yang" within the body. This case report highlights the importance of medical education in cultural competency. Additional barriers due the COVID19 pandemic, such as physical distancing, use of PPE, and telehealth services, challenge the development of rapport with a patient by hindering direct physician-patient communication, creating difficulty in patient interview and history taking. Though chrysanthemum flower and peppermint, to name a few herbal treatments, may not be considered therapeutic in Western medicine, they are common in TCM for a variety of disease presentations. A healthcare provider with understanding of TCM customs can elicit more open communication, retrieve more detailed history, develop a better rapport, create a stronger physician-patient relationship, improve patient compliance, and potentially lessen unnecessary testing. Thus, cultural competency training for healthcare providers can thus result in improved patient care during the COVID-19 pandemic through the recognition and acknowledgment of customs.

\section{References}

[1]. Tang J-L, Liu B-Y, Ma K-W: Traditional Chinese medicine. The Lancet. 2008, 372:1938-1940. 10.1016/S01406736(08)61354-9

[2]. Zhao X, Tan X, Shi H, Xia D: Nutrition and traditional Chinese medicine (TCM): a system's theoretical perspective. European Journal of Clinical Nutrition. 2020, 75:1-7. 10.1038/s41430-020-00737-w

[3]. Chin J, Li S, Yim G, Zhou YA, Wan PJ, Dube ER, Volokitin M, Sahni S, Terrell MA, Lomiguen CM: Perceptions of the osteopathic profession in New York City's Chinese Communities. Fam Med Community Health. 2020, 8:e000248. 10.1136/fmch-2019-000248

[4]. Chin J, Woo H, Choi D, Dube E, Volokitin M, Lomiguen C: Perceptions of the Osteopathic Profession in New York City's Korean Communities. Osteopathic Family Physician. 2020, 13. 10.33181/13011

[5]. Amin S, Chin J, Terrell MA, Lomiguen CM: Addressing Challenges in Humanistic Communication During COVID-19 Through Medical Education. Frontiers in Communication. 2021, 6 . 10.3389/fcomm.2021.619348 
[6]. Lomiguen CM, Rosete I, Chin J: Providing Culturally Competent Care for COVID-19 Intensive Care Unit Delirium: A Case Report and Review. Cureus. 2020, 12:e10867. 10.7759/cureus. 10867

[7]. Hou Y, Jiang JG: Origin and concept of medicine food homology and its application in modern functional foods. Food Funct. 2013, 4:1727-1741. 10.1039/c3fo60295h

[8]. Jiang S, Quave CL: A comparison of traditional food and health strategies among Taiwanese and Chinese immigrants in Atlanta, Georgia, USA. J Ethnobiol Ethnomed. 2013, 9:61. 10.1186/1746-4269-9-61

[9]. Kong $\mathrm{H}$, Hsieh $\mathrm{E}$ : The social meanings of traditional Chinese medicine: elderly Chinese immigrants' health practice in the United States. J Immigr Minor Health. 2012, 14:841849. 10.1007/s10903-011-9558-2

[10]. Zou P: Traditional Chinese Medicine, Food Therapy, and Hypertension Control: A Narrative Review of Chinese Literature. Am J Chin Med. 2016, 44:1579-1594. 10.1142/S0192415X16500889

[11]. Chen Q, Wang Q, Ding S, Li S, Zhang Y, Chen $\mathrm{S}$, Lin X, Li C, Asakawa T: Problems lowering the study quality in traditional medicine, introspection from an example of metaanalysis of acupuncture. BMC Complement Med Ther. 2020, 20:41. 10.1186/s12906-0192806-z

[12]. Wang Q, Su CP, Zhang HM, Ren YL, Wang W, Guo SZ: [Anti-inflammatory mechanism of heat-clearing and detoxifying Chinese herbs]. Zhongguo Zhong Yao Za Zhi. 2018, 43:37873794. 10.19540/j.cnki.cjcmm.20180611.012

[13]. Lam P, Cheung F, Tan HY, Wang N, Yuen MF, Feng Y: Hepatoprotective Effects of Chinese Medicinal Herbs: A Focus on AntiInflammatory and Anti-Oxidative Activities. Int J Mol Sci. 2016, 17:465. 10.3390/ijms 17040465

[14]. Chandrasekara A, Shahidi F: Herbal beverages: Bioactive compounds and their role in disease risk reduction - A review. $J$ Tradit Complement Med. 2018, 8:451-458. 10.1016/j.jtcme.2017.08.006

[15]. Xiao J, Yang Y, Zhu Y, Qin Y, Li Y, Fu M, Zhai Z, Zhu L: Efficacy and Safety of Traditional Chinese Medicine on Nonerosive Reflux Disease: A Meta-Analysis of Randomized Controlled Trials. Evid Based Complement
Alternat Med. 2018, 2018:1505394. 10.1155/2018/1505394

[16]. Dai YK, Wu YB, Wen H, Li RL, Chen WJ, Tang C, Lu L, Hu L: Different Traditional Herbal Medicines for the Treatment of Gastroesophageal Reflux Disease in Adults. Front Pharmacol. 2020, 11:884. 10.3389/fphar.2020.00884

[17]. Wang WJ, Zhang T: Integration of traditional Chinese medicine and Western medicine in the era of precision medicine. J Integr Med. 2017, 15:1-7. 10.1016/S2095-4964(17)603145

[18]. Naito T, Chin J, Lin J, Shah PJ, Lomiguen CM: Postpartum psychosis in a non-native language-speaking patient: A perspective on language barriers and cultural competency. Gen Psychiatr. 2019, 32:e100077. 10.1136/gpsych-2019-100077

[19]. Naito T, Chin J, Kim TU, Veera S, Jeannette M, Lomiguen CM: Further Reduction in HelpSeeking Behaviors Amidst Additional Barriers to Mental Health Treatment in Asian Populations: A Contemporary Review. Cureus. 2020, 12:e11455. 10.7759/cureus.11455

[20]. Lin JH, Chin, J., Yu, Z.L., Kviatkovsky, B., \& Lomiguen, C.M.: Benzodiazepine Overdose in a Non-English-Speaking Patient: Case Report on Cultural Competency and Asian Pharmacy Practices. International Journal of Psychiatry Research. 2019, 2:1-3. 